\title{
The Role of Cellular Immunity Both in the Induction and Effector Phases of Experimental Allergic Blepharoconjunctivitis (EAC) in Rats
}

\author{
A. FUKUSHIMA*, H. YOSHIDA, H. IWAMOTO, O. YOSHIDA AND H. UENO \\ Laboratory of Immunology, Department of Ophthalmology, Kochi Medical School, Kohasu, Oko-cho, \\ Nankoku 783, Japan
}

(Received Columbia 21 January 1997 and accepted in revised form 12 June 1997)

\begin{abstract}
In allergic conjunctivitis, the early phase reaction has been studied extensively both in humans and animals. Although cellular infiltration is the main feature of the late phase reaction, the role of cellular immunity remains unclear. The purpose of this study was to elucidate the role of cellular immunity both in the induction and effector phases of experimental allergic blepharoconjunctivitis (EAC). To analyse the involvement of cellular immunity in the induction phase, 6-8-week-old male Lewis rats were immunized with ovalbumin (OVA) emulsified with complete Freund's adjuvant (CFA), incomplete Freund's adjuvant $(\mathrm{IFA})$, TiterMax ${ }^{\mathrm{R}}(\mathrm{TM})$, aluminum hydroxide $\left[\mathrm{AI}(\mathrm{OH})_{3}\right]$, or without any adjuvant. Three weeks after immunization, the rats were challenged with OVA by eye drops, and $24 \mathrm{hr}$ later they were euthanized and their eyes, including the lids, blood, and lymph nodes were harvested for analysis of disease and immune responses. The results indicated that adjuvants were necessary to induce disease as well as both cellular and humoral immunity. $\mathrm{Al}(\mathrm{OH})_{3}, \mathrm{CFA}$ and $\mathrm{TM}$ induced stronger disease and cellular immunity than IFA. The intensity of disease correlated with that of cellular immunity. To further investigate the involvement of cellular immunity in EAC, lymph node cells collected from immunized rats were adoptively transferred into naive syngeneic recipients that were challenged 4 days later with OVA. EAC developed in the recipients of lymph node cells that were also stimulated in culture with OVA. These recipient rats developed cellular infiltration in the lid and conjunctiva, in a dose-dependent manner. These results suggest that cellular immunity played a major role in the development of EAC, both in the induction and effector phases. (C) 1997 Academic Press Limited

Key words: allergy; conjunctivitis; adjuvant; adoptive transfer; cellular immunity; rats; EAC.
\end{abstract}

\section{Introduction}

Allergic conjunctivitis is one of the most common ocular pathological conditions evaluated in eye clinics. Chief complaints of patients include itching and burning, and their clinical signs are chemosis, conjunctival hyperemia and lid swelling (Tuft et al., 1991). Studies of experimental animal models which closely resemble human allergic conjunctivitis indicated that IgE mediated type I allergy is mainly involved in the induction of this form of the disease (Donshik, 1988; Doherty, 1989; Anderson, 1992).

During the development of allergy, two phases, namely, early phase reaction and late phase reaction are observed (Gleich, 1982). Early phase reaction is due to upregulation of vascular permeability caused by agents such as histamines (Kitamura, 1989), and late phase reaction is induced by chemoattractant factor-dependent cellular infiltration (Zweiman, 1993). Early phase reaction in allergy has been studied extensively and therapeutic approaches targeted for it, such as topical vasoconstrictor/

\footnotetext{
* For correspondence at: Atsuki Fukushima, Laboratory of Immunology, Department of Ophthalmology, Kochi Medical School, Kohasu, Oko-cho, Nankoku 783, Japan.
}

antihistamine agents, are being used in clinics (Norman, 1993).

In the initiation of immune responses in experimental animals, adjuvants have been used to augment either cellular immunity, humoral immunity, or both (Coligan et al., 1992; Paul, 1993). Immunization with a protein in the absence of adjuvants can elicit a much weaker or diminished immunity. It is generally thought that adjuvants such as CFA tend to promote higher Th1 cell induction than Th2, whereas those as IFA tend to promote higher Th2 cell induction (Germann et al., 1995; Mauri et al., 1996; Trinchieri, 1995). Therefore, it may be possible to manipulate the immune status using a particular adjuvant.

Recently, the mechanism of cellular infiltration has been studied particularly with regard to asthma and the essential role of T-cells in chronic asthma was suggested by J. G. Martin and his coworkers (Olivenstein et al., 1993; Watanabe et al., 1995). This group reported that adoptive transfer of antigen primed CD4 positive T-cells could induce allergic airway responses and analyzed the detailed mechanisms of their roles in late phase reaction.

Based on this information, we investigated the role of four adjuvants in cellular immune responses in EAC and whether EAC could be transferred into naive 
recipients by adoptive transfer of immune cells. We also tested the effects of stimulation in vitro on adoptive transfer of both disease and immunity.

\section{Materials and Methods}

Rats

Six- to eight-week-old male Lewis rats were purchased from a commercial source (Seac Yoshitomi, Fukuoka, Japan) and maintained in a specific pathogen-free animal facility at Kochi Medical School. All animal procedures conformed to Institutional Guidelines and the ARVO Resolution on Use of Animals in Research.

\section{Immunization}

Rats were injected with $100 \mu \mathrm{g}$ of ovalbumin (OVA, grade V, Sigma Immunochemicals, St. Louis, MO, U.S.A.), with or without adjuvant, in a final volume of $100 \mu \mathrm{l}$ in their left hind foot-pad. The adjuvants used were: CFA (Yatoron, Tokyo, Japan), IFA, TM (CytRx Co., Atlanta, GA) (Roberge et al., 1992) and $\mathrm{Al}(\mathrm{OH})_{3}$ $(200 \mathrm{mg} / \mathrm{ml})$.

\section{Experimental Protocol}

(1) Optimization of experimental conditions: CFA or TM was chosen for the adjuvant in this experiment. To find out the best time for challenge, the rats received eye drops in both conjunctival sacs with $250 \mu \mathrm{g}$ of OVA in $50 \mu \mathrm{l}$ phosphate-buffered saline (PBS) at $7,14,21$ or 28 days after immunization. Twenty four hours later, they were clinically examined. Then, they were killed by diethylether and the eyes including lids, blood and lymph nodes were harvested for histology, antibody titer by enzymelinked immunosorbent assay (ELISA) and lymphocyte proliferation assay, respectively. The detailed methods are described below. To observe the clinical changes after challenge, OVA-primed rats were examined clinically at 6,24 or $48 \mathrm{hr}$ after challenge.

(2) Analysis of effects of the four adjuvants on the induction phase by active immunization: 21 days after immunization with or without any adjuvant, rats were challenged with OVA as described above. Twenty four hours later, the rats were killed and the same procedures as (1) were performed.

(3) Analysis of effector phase by passive immunization (adoptive transfer of OVA primed cells) : cells from immune inguinal lymph nodes were harvested 14 days after immunization. The cells were washed three times with RPMI 1640 medium (Nikken Bio Medical Laboratory, Kyoto, Japan) supplemented with 100 $\mathrm{U} / \mathrm{ml}$ penicillin, $100 \mu \mathrm{g} / \mathrm{ml}$ streptomycin and $2 \mathrm{mM} \mathrm{L-}$ glutamine and injected to naive syngeneic rats directly or following stimulation in culture. For the stimu- lation, OVA primed lymph node cells $\left(100 \times 10^{6}\right)$ were cultured in $25 \mathrm{~cm}^{2}$ flasks (Nunc, Roskilde, Denmark) in $10 \mathrm{ml}$ RPMI 1640 supplemented with $2 \mathrm{mM} \mathrm{L-}$ glutamine, $5 \times 10^{-5}$ M 2-mercaptoethanol (2-ME), 100 $\mathrm{U} / \mathrm{ml}$ penicillin, $100 \mu \mathrm{g} / \mathrm{ml}$ streptomycin, $50 \mu \mathrm{g} / \mathrm{ml}$ OVA and containing $10 \%$ fetal calf serum (FCS, ICN Biomedical Japan Co., Tokyo, Japan). After incubation for 3 days, stimulated lymph node cells were harvested, washed three times, and injected into recipient rats intravenously (Mochizuki et al., 1985). Four days following the cell injection, recipients were challenged with OVA as described above. Eyes, blood and spleens were harvested $24 \mathrm{hr}$ after challenge for histology, ELISA, and proliferative responses, respectively.

(4) Analysis of effector phase by adoptive transfer of immune serum: serum from immunized rats were collected and pooled 21 days after immunization. Their antibody titers against OVA were tested by ELISA. (1 ml) was injected intravenously into naive rats and they were challenged just after transfer. The animals were examined for the development of EAC every $6 \mathrm{hr}$ until $24 \mathrm{hr}$ after transfer at which time the animals were killed.

\section{Clinical Grading and Cell Counting}

The following clinical scoring system was used. Each eye was assessed for the findings of lid swelling and discharge, and each finding was scored as 1 point. For example, if the right eye had no changes and the left eye had both discharge and lid swelling, the grading score of this rat was 2 points. Ocular changes were also examined histologically. After clinical evaluation, eyes, including the lids, were fixed in 10\% buffered formalin and embedded in paraffin. Sections $(10 \mu \mathrm{m})$ were cut and stained with hematoxylin-eosin. The infiltrated cells were counted in the palpebral conjunctiva using an eye piece grid at $400 \times$ magnification.

\section{Assessment of Cellular Immune Responses}

In each experiment, either lymph nodes or spleens from two rats were combined per group and experiments were repeated as recorded. Lymph node cells were used for experiments of active immunization, and splenocytes for those of passive immunization. Lymphocyte proliferation assays were set up in quadruplicate in 96-well flat bottom plates. Either lymph node cells or splenocytes $\left(3 \times 10^{5}\right.$ cells $/$ well $)$ were cultured in a final volume of $0.2 \mathrm{ml}$ RPMI 1640 medium supplemented with $5 \%$ FCS, 2-ME $\left(5 \times 10^{-5} \mathrm{M}\right)$, Lglutamine $(2 \mathrm{~mm})$, penicillin $(100 \mathrm{U} / \mathrm{ml})$ and streptomycin $(100 \mu \mathrm{g} / \mathrm{ml})$. Cells were stimulated with OVA at final concentrations of $0 \cdot 1,1,10,100 \mu \mathrm{g} / \mathrm{ml}$. After incubation for $72 \mathrm{hr}$ at $37^{\circ} \mathrm{C}$ in a humidified atmosphere with $5 \% \mathrm{CO}_{2}$, cultures were pulsed for $16 \mathrm{hr}$ with $0.5 \mu \mathrm{Ci} /$ well of $\left[{ }^{3} \mathrm{H}\right]$ thymidine (Japan Atomic Energy Research Institute, Tokai, Japan). Cultures 
were then harvested and the radioactivity was measured by standard techniques. Data were expressed as stimulation indices (S.I.).

\section{Assessment of Humoral Immune Responses}

Serum was collected from each rat via cardiac puncture at the time of killing. Serum antibody levels against OVA were measured by direct ELISA. OVA ( $500 \mathrm{ng} /$ well) were absorbed to 96 -well plates for $2 \mathrm{hr}$ at $37^{\circ} \mathrm{C}$. The plates were washed with PBS-Tween and serially diluted serum samples were added to the wells. Bound $\mathrm{Ab}$ was detected by peroxidase-conjugated anti-IgG or IgE (Nordic Immunological Laboratories, Tilburg, The Netherlands). The plates were developed using 3,3',5,5'-tetramethylbenzidine base (TMBELISA, GIBCO BRL, Gaithersburg, MD, U.S.A.) and the optical density (OD) for each well was read at $610 \mathrm{~nm}$.

\section{Results}

\section{Time-course of EAC}

The time-course of EAC in rats immunized with OVA emulsified with CFA or TM is shown in Table I. As seen, the onset of EAC was at day 14 after immunization and the inflammation continued until day 28. The maximal grade was obtained at day 21 . These results were further supported by the histological examination which showed cellular infiltration in the lid and conjunctiva. The correlation of clinical score and histological findings was summarized in Table II. Interestingly, even though there were

TABLE I

Disease grading of immunized rats: Time course after immunization

\begin{tabular}{lllll}
\hline $\begin{array}{l}\text { Days after } \\
\text { immunization }\end{array}$ & \multicolumn{1}{c}{7} & \multicolumn{1}{c}{14} & 21 & 28 \\
\hline OVA/CFA & $0 / 6^{*}$ & $6 / 6$ & $6 / 6$ & $6 / 6$ \\
& $(0 \cdot 0) \dagger$ & $(2 \cdot 7)$ & $(4 \cdot 0)$ & $(2 \cdot 5)$ \\
OVA/TM & $0 / 6$ & $4 / 6$ & $6 / 6$ & $6 / 6$ \\
& $(0 \cdot 0)$ & $(1 \cdot 7)$ & $(4 \cdot 0)$ & $(3 \cdot 5)$ \\
\hline
\end{tabular}

* The number of rats developed EAC/the number of rats tested. $\dagger$ The mean grading score followed by the grading system written in materials and methods.

TABLE II

Correlation between clinical grading and cell counting

\begin{tabular}{cc}
\hline $\begin{array}{c}\text { Clinical } \\
\text { grading }\end{array}$ & $\begin{array}{c}\text { Infiltrated cell number } \\
\text { (mean } \pm \text { s.E.) }\end{array}$ \\
\hline 0 & $4 \cdot 2 \pm 0 \cdot 5(n=10)^{*}$ \\
2 & $107 \pm 21 \cdot 3(n=8)$ \\
4 & $312 \pm 171 \cdot 1(n=24)$ \\
\hline
\end{tabular}

\footnotetext{
* Number of recipients tested.
}

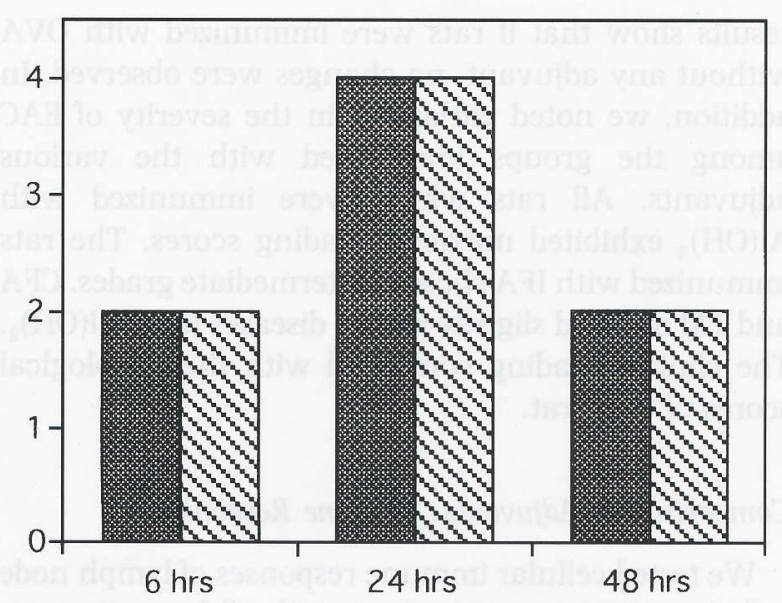

FIG. 1. Clinical grading of EAC in OVA immunized rats

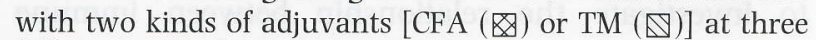
different time points after challenge.

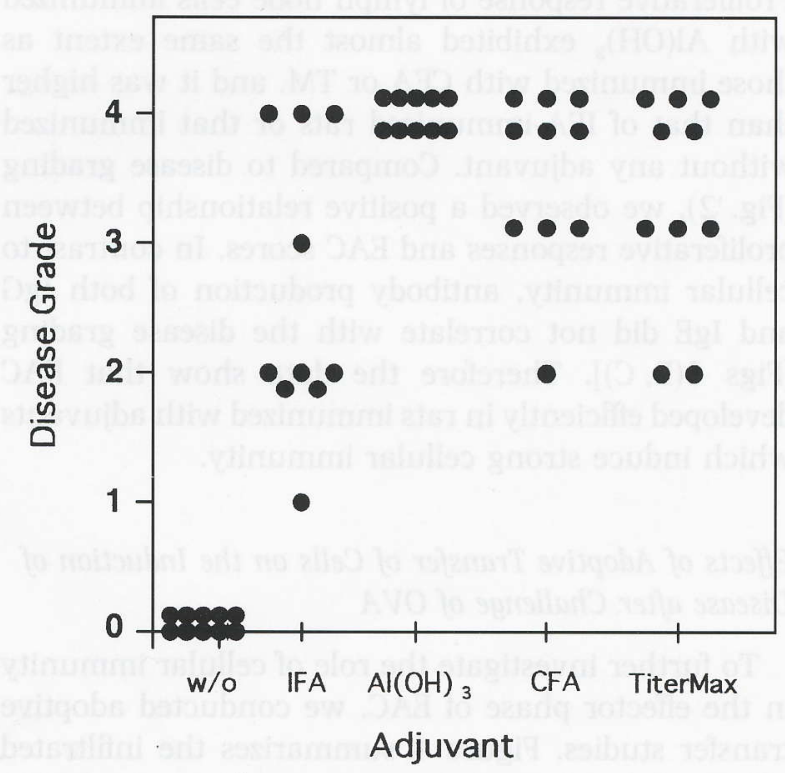

FIG. 2. Clinical grading of EAC in OVA immunized rats with four kinds of adjuvants or without any adjuvants. Twenty-one days after immunization, Lewis rats were challenged with OVA by eye drops, and they were checked and graded clinically $24 \mathrm{hr}$ later, following materials and methods.

no remarkable differences in disease between the two groups, the proliferative responses of lymph node cells from the TM group started to be observed 7 days after immunization (data not shown). We also tested the kinetics of disease grade after challenge from $6 \mathrm{hr}$ to $48 \mathrm{hr}$ after eye drops. As shown in Fig. 1, cellular infiltration began to be noticed $24 \mathrm{hr}$ after challenge and continued until $48 \mathrm{hr}$. From these results, we chose day 21 for the challenge and $24 \mathrm{hr}$ after challenge as the time point of killing.

\section{Comparison of Adjuvants: Induction of EAC}

The clinical grading of EAC in rats immunized with the different adjuvants is summarized in Fig. 2. The 
results show that if rats were immunized with OVA without any adjuvant, no changes were observed. In addition, we noted variations in the severity of EAC among the groups immunized with the various adjuvants. All rats which were immunized with $\mathrm{Al}(\mathrm{OH})_{3}$ exhibited maximal grading scores. The rats immunized with IFA showed intermediate grades. CFA and TM induced slightly milder diseases than $\mathrm{Al}(\mathrm{OH})_{3}$. The clinical grading correlated with the histological scores of each rat.

\section{Comparison of Adjuvants: Immune Responses}

We tested cellular immune responses of lymph node cells from OVA immunized rats with all four adjuvants to investigate the relationship between immune responses and the development of EAC. Figure 3(A) shows the cellular proliferative responses in vitro. Proliferative response of lymph node cells immunized with $\mathrm{Al}(\mathrm{OH})_{3}$ exhibited almost the same extent as those immunized with CFA or TM, and it was higher than that of IFA-immunized rats or that immunized without any adjuvant. Compared to disease grading (Fig. 2), we observed a positive relationship between proliferative responses and EAC scores. In contrast to cellular immunity, antibody production of both IgG and IgE did not correlate with the disease grading [Figs $3(\mathrm{~B}, \mathrm{C})]$. Therefore the data show that EAC developed efficiently in rats immunized with adjuvants which induce strong cellular immunity.

\section{Effects of Adoptive Transfer of Cells on the Induction of Disease after Challenge of OVA}

To further investigate the role of cellular immunity in the effector phase of EAC, we conducted adoptive transfer studies. Figure 4 summarizes the infiltrated cell numbers of recipient groups which received OVA primed lymph node cells with or without activation in vitro. In both groups, the infiltrated cell number is related to the cell number which was adoptively transferred. A comparison of the intensity of disease between recipients of stimulated cells and unstimulated cells showed that stimulation in vitro enhanced dramatically the lymphocyte capacity to adoptively transfer the disease whereas unstimulated cells had minimal or no activity.

\section{Immunity in Recipients of OVA Primed Cells}

Our data suggest that cellular immunity is related to the severity of disease in the induction phase. To further analyse the roles of cellular immunity in the effector phase, proliferation assays and ELISA were performed with splenocytes and sera of the recipient rats. No proliferative responses were detected in most recipient rats with the exception of the group that received $50 \times 10^{6}$ stimulated cells [Fig. 5(A)]. Low levels of IgG antibody production were found in most recipients [Fig. 5(B)]. The amplitude of Ab production is dependent on the transferred cell number. $\operatorname{IgE}$ was not detected in any recipients in our study (data not shown).

\section{Analysis of Recipients of OVA Antibodies}

To compare the involvement of cellular immunity with humoral immunity in the development of disease, pooled serum from OVA-immunized rats was injected into naive recipient rats. The recipients were challenged and euthanized at various time intervals. Even though they developed edema in conjunctiva and lid, no cellular infiltration was observed (data not shown).

\section{Discussion}

Generally speaking, allergic pathological conditions, such as asthma, rhinitis or conjunctivitis are thought to be dominantly caused by IgE-mediated humoral immunity as the initial step. Recently, several groups suggested that cellular immunity may be the inducing mechanism of the allergic changes. For example. Martin's group showed that antigen specific CD $4^{+} \mathrm{T}$ cells could transfer asthma in an experimental animal model (Olivenstein et al., 1993; Watanabe et al., 1995). These studies indicate that not only humoral but also cellular immunity, especially T-cell-mediated immunity, is actively involved in the induction and effector phases of allergic reactions.

Although it is well known that genetic factors are important in the induction of allergy, little is known concerning other elements which influence the induction of allergy. In experimental models, adjuvants are usually used for effective induction of immune responses. It is also known that some adjuvants tend to induce cellular immunity, while others enhance humoral responses (Germann et al., 1995; Trinchieri, 1995; Mauri et al., 1996). If we could manipulate immune status toward either cellular or humoral by changing adjuvants, comparison of adjuvants will give us information on which immunity is mainly involved in the induction of disease. These findings led us to investigate the roles of adjuvants in the induction of EAC.

The intensity of disease was compared using four different adjuvants. It is clear that adjuvants are necessary for the induction of disease (Fig. 2). In our system, immunization was performed routinely only once. Yet, no disease or immune response were detected in rats immunized with OVA solution without any adjuvant five times repeatedly (data not shown). These studies thus further indicate the essential role of adjuvants in the development of EAC.

We also noted a difference concerning the effectiveness of disease induction among the four adjuvants. Rats immunized with IFA demonstrated less severe disease than those with CFA, TM or $\mathrm{Al}(\mathrm{OH})_{3}$ (Fig. 2). It is of note that there seems to be a relationship 

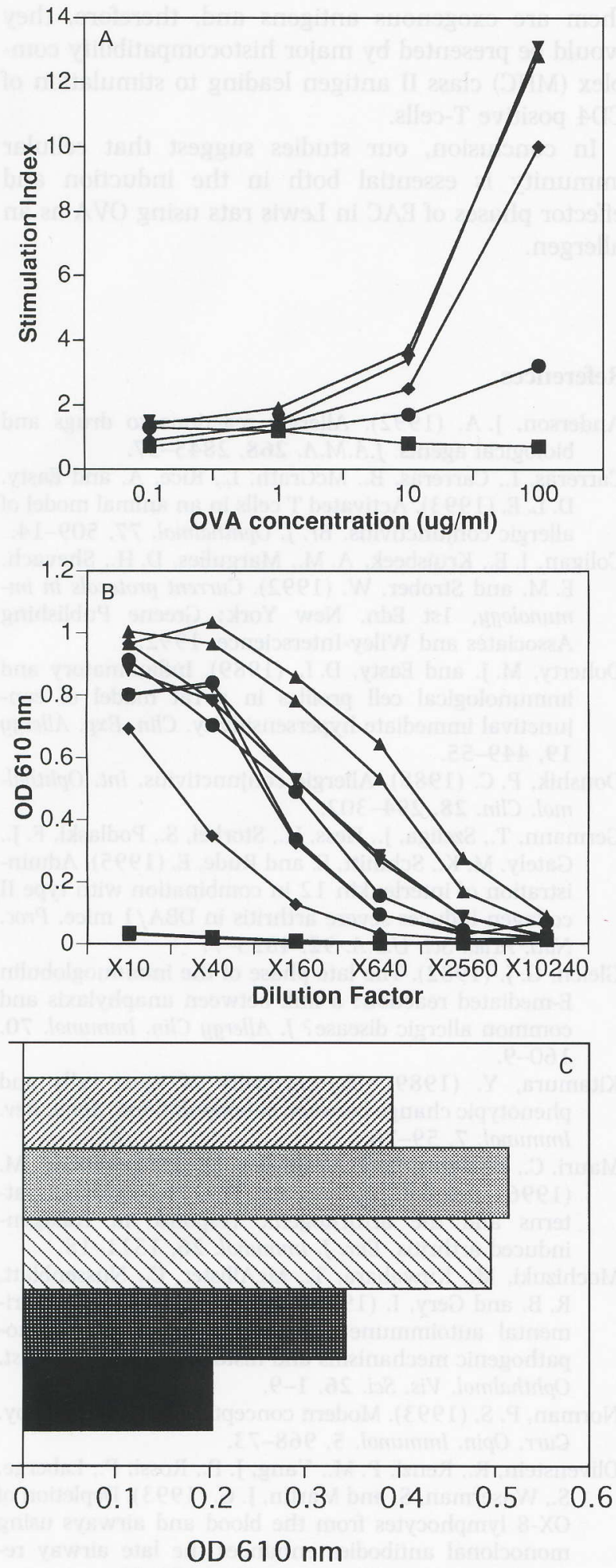

FIG. 3. (A) Proliferative response by LN cells of Lewis rats immunized with OVA in four kinds of adjuvants or without any adjuvant. Draining LN cells were harvested and tested for proliferation detailed in materials and methods. The recorded data are means of S.I. values of two rats of each group. Similar results were obtained in four more independent experiments. The combined mean cpm values in the unstimulated control cultures of rats of different adjuvants were between 139 and 503. ( $\square$ ), without adjuvant; $(\bullet), \mathrm{Al}(\mathrm{OH})_{3} ;(\mathbf{\Delta}), \mathrm{CFA} ;(\mathbf{O}), \mathrm{IFA} ;(\boldsymbol{\nabla})$, TM. (B) Measurement of OVA specific IgG by ELISA in serum from Lewis rats immunized with OVA in four kinds of adjuvants or without any adjuvants. Serum was collected at the time

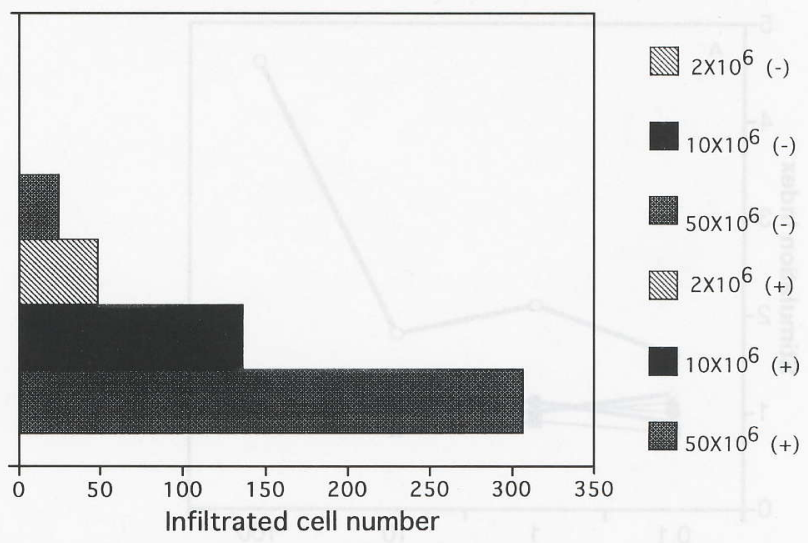

Fig. 4. Infiltrated cell number in the conjunctival fornix of recipients of OVA-primed cells either stimulated in vitro $(+)$ or not $(-)$. Recipients were transferred with 2, 10 or $50 \times 10^{6}$ OVA primed LN cells. Four days later, they were challenged with OVA by eye drops and eyes, including the lids, were harvested for histology $24 \mathrm{hr}$ after challenge. $x$-axis indicates the mean number of infiltrated cells of six rats.

between the grade of disease and cellular proliferative responses in vitro in each group of adjuvant [Fig. 2, 3(A)], thus suggesting that cellular immunity is dominantly involved in the induction of disease in our model. It is necessary to study cytokine production both in vivo and in vitro to elucidate the exact involvement of cellular immunity in the induction of EAC.

Carreras et al. (1993) reported that activated Tcells, which are specific to antigen and infiltrate the conjunctiva, play an important role in the pathogenesis of allergic conjunctivitis. To clarify the role of T-cells in the induction of EAC, we have employed the system of adoptive transfer of activated T-cells into naive recipients. We transferred lymph node cells from immunized rats, either stimulated in vitro or not, into naive syngeneic recipients. Recipient rats demonstrated cellular infiltration in the conjunctiva and lid following a challenge with OVA. The infiltrated cell number was dependent on the transferred cell number and stimulation in vitro of the injected cells. This is the first report to show that EAC is transferable by immune cells and cellular immunity is actively involved in the effector phase of development of this disease.

In contrast to the efficient adoptive transfer of

of killing and assayed for ELISA. $x$-axis indicates dilution factors of serum, and $y$-axis depicts absorbance at $610 \mathrm{~nm}$. Similar results were obtained in four more independent experiments. Symbol meaning are same as part (A). (C) Measurement of OVA specific IgE by ELISA in serum from Lewis rats immunized with OVA in four kinds of adjuvants or without any adjuvant. Dilution factor in this experiment is $\times 40, x$-axis indicates absorbance at $610 \mathrm{~nm}$. Similar results were obtained in four more independent experiments.

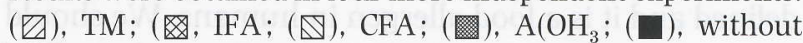
adjuvant. 

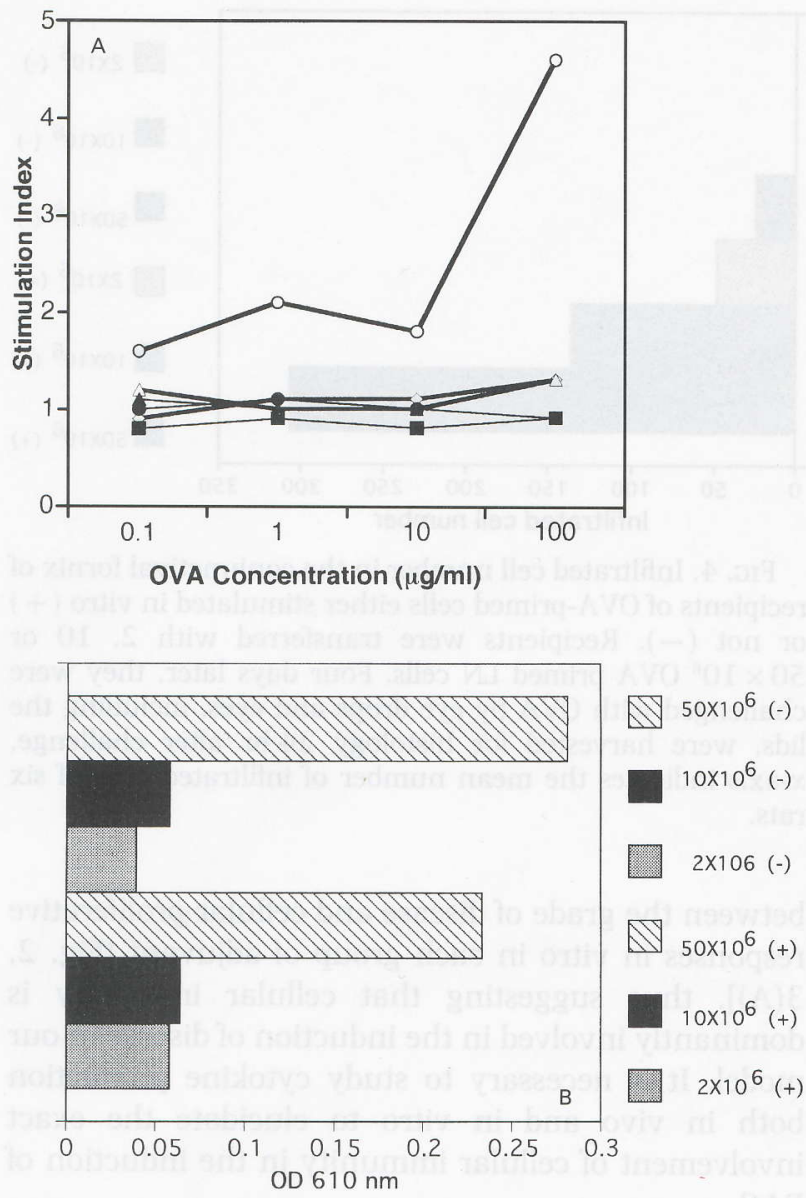

FIG. 5. (A) Proliferative responses of spleen cells from recipients transferred with OVA primed LN cells either stimulated in vitro (filled symbols) or not (open symbols). Recipients were transferred with $2(\diamond, \diamond), 10(\Delta, \triangle)$ or $50 \times 10^{6}(0)$ OVA-primed LN cells. Four days later, they were challenged with OVA by eye drops and spleens were harvested $24 \mathrm{hr}$ after challenge to examine proliferative responses. The recorded data are means of S.I. values of two rats of each group. Similar results were obtained in two more independent experiments. The combined mean $\mathrm{cpm}$ values in the unstimulated control cultures of rats of different adjuvants were between 1420 and 3524. (B) Measurement of OVA specific IgG by ELISA in serum from recipients transferred with OVA primed LN cells. Serum was collected at the time of killing and assayed for ELISA. $x$-axis indicates the absorbance at $610 \mathrm{~nm}$ (dilution factor is $\times 40$ ). Similar results were obtained in two more independent experiments.

disease by cells, we failed to induce cellular infiltration by adoptive transfer of immune serum. This result is in accordance with the report of Sorkness and coworkers (1988), that adoptive transfer of a monoclonal IgE antibody could not induce late phase reaction in an allergic asthma model.

Although we used only OVA as an allergen in EAC, it is obviously compatible to use ragweed or dermatophagoid antigens to analyse our model for human allergic conjunctivitis. OVA is used for an allergen in experimental allergic models of not only conjunctivitis but also other diseases such as asthma, since it is well defined and it is a food allergen in humans. We should conduct experiments using other antigens, but all of them are exogenous antigens and, therefore, they would be presented by major histocompatibility complex (MHC) class II antigen leading to stimulation of CD4 positive T-cells.

In conclusion, our studies suggest that cellular immunity is essential both in the induction and effector phases of EAC in Lewis rats using OVA as an allergen.

\section{References}

Anderson, J. A. (1992). Allergic reactions to drugs and biological agents. J.A.M.A. 268, 2845-57.

Carreras, I., Carreras, B., McGrath, L., Rice, A. and Easty, D. L. E. (1993). Activated T cells in an animal model of allergic conjunctivitis. Br. J. Ophthalmol. 77, 509-14.

Coligan, J. E., Kruisbeek, A. M., Margulies, D. H., Shevach, E. M. and Strober, W. (1992). Current protocols in immunology, 1st Edn. New York: Greene Publishing Associates and Wiley-Interscience, 1992.

Doherty, M. J. and Easty, D. L. (1989). Inflammatory and immunological cell profiles in a rat model of conjunctival immediate hypersensitivity. Clin. Exp. Allergy 19, 449-55.

Donshik, P. C. (1988). Allergic conjunctivitis. Int. Ophthalmol. Clin. 28, 294-302.

Germann, T., Szeliga, J., Hess, H., Storkel, S., Podlaski, F. J., Gately, M. K., Schmitt, E. and Rude, E. (1995). Administration of interleukin 12 in combination with type II collagen induces severe arthritis in DBA/1 mice. Proc. Natl. Acad. Sci. U.S.A. 92, 4823-7.

Gleich, G. J. (1982). The late phase of the immunoglobulin E-mediated reaction: a link between anaphylaxis and common allergic disease? J. Allergy Clin. Immunol. 70 , $160-9$.

Kitamura, Y. (1989). Heterogeneity of mast cells and phenotypic change between subpopulations. Annu. Rev. Immunol. 7, 59-76.

Mauri, C., Williams, R. O., Walmsley, M. and Feldmann, M. (1.996). Relationship between Th1/Th2 cytokine patterns and the arthritogenic response in collageninduced arthritis. Eur. J. Immunol. 26, 1511-18.

Mochizuki, M., Kuwabara, T., McAllister, C., Nussenblatt, R. B. and Gery, I. (1985). Adoptive transfer of experimental autoimmune uveoretinitis in rats: immunopathogenic mechanisms and histologic features. Invest. Ophthalmol. Vis. Sci. 26, 1-9.

Norman, P. S. (1993). Modern concepts of immunotherapy. Curr. Opin. Immunol. 5, 968-73.

Olivenstein, R., Renzi, P. M., Yang, J. P., Rossi, P., Laberge, S., Waserman, S. and Martin, J. G. (1993). Depletion of OX-8 lymphocytes from the blood and airways using monoclonal antibodies enhances the late airway response in rats. J. Clin. Invest. 92, 1477-82.

Paul, W. E. (1993). Fundamental immunology, 3rd Edn. New York: Raven Press, 1993.

Roberge, F. G., Xu, D. and Chan, C.-C. (1992). A new effective and non-harmful chemical adjuvant for the induction of experimental autoimmune uveoretinitis. Curr. Eye Res. 11, 371-6.

Sorkness, R., Blythe, S. and Lemanske, R. F. Jr (1988). Pulmonary antigen challenge in rats passively sensitized with a monoclonal IgE antibody induces immediate but not late changes in airway mechanics. Am. Rev. Respir. Dis. 138, 1152-6.

Trinchieri, G. (1995). Interleukin-12: a proinflammatory 
cytokine with immunoregulatory functions that bridge innate resistance and antigen-specific adaptive immunity. Annu. Rev. Immunol. 13, 251-76.

Tuft, S. J., Kemeney, D. M., Dart, J. K. G. and Buckley, R. J. (1991). Clinical features of atopic keratoconjunctivitis. Ophthalmology 98, 150-8.
Watanabe, A., Mishima, H., Renzi, P. M., Xu, L.-J., Hamid, Q. and Martin, J. G. (1995). Transfer of allergic airway responses with antigen-primed $\mathrm{CD} 4+$ but not $\mathrm{CD} 8+\mathrm{T}$ cells in Brown Norway rats. J. Clin. Invest. 96, 1303-10.

Zweiman, B. (1993). The late-phase reaction: role of IgE, its receptor and cytokines. Curr. Opin. Immunol. 5, 950-5. 